\title{
TRAITEMENT DU CANCER DU TESTICULE
}

\author{
Pr Marcel Krulik* \\ * Chef de Service de Médecine interne - Oncologie - Hôpital Saint Antoine \\ 184, rue du Faubourg Saint-Antoine - 75571 PARIS Cedex
}

\section{TESTICULAR CANCER TREATMENT.}

Significant advances have been achieved in testicular cancer treatment for the last 15 years. Almost $100 \%$ of the non seminomatous tumors seen at the precocious stages I and II are cured because of the efficacy of chemotherapy in relapses. For clinical stages I, tendancy is to survey after castration without lymphadenectomy. For advanced metastatic stages, platinum has changed chemotherapy performances, allowing to cure 75 to $80 \%$ of these patients. The results of a bad pronosis group with large tumor remain to be improved. Seminomas at a localized stage (the most frequent case) are cured by radiotherapy; at an advanced stage, they are as sensitive to chemotherapy as non seminomatous tumors. Key-words : seminoma, non seminomatous tumor, chemotherapy, radiotherapy, surgery, prognosis, staging. Andrologie, 1991, 1:32-33

Le cancer du testicule est la malignité la plus fréquente chez l'homme de 35-45 ans. Des progrés considérables ont été accomplis qui ont permis de réduire de plus de $50 \%$ la mortalité par cette affection (8). Il s'agit presque toujours de tumeurs germinales réparties en deux catégories : les séminomes $(40 \%)$ et les tumeurs non séminomateuses (carcinome embryonnaire, tumeur du sac vitellin, tératome, choriocarcinome, association de ces types histologiques entre eux et/ou avec les séminomes).

TUMEURS NON SEMINOMATEUSES (TNS) I - La stratégie thérapeutique repose sur le staging

a) La classification la plus employée est celle du Royal Marsden Hospital (5)

- Stade I : Tumeur limitée au testicule (IM + = Beta $\mathrm{HCG}+$, ou AFP +)

- Stade II : Atteinte ganglionnaire abdominale $\mathrm{A}<2 \mathrm{~cm}$; B 2-5 cm; C : $>5 \mathrm{~cm}$

- Stade III : Atteinte ganglionnaire susdiaphragmatique

- Stade IV : Métastases extra ganglionnaires

- pulmonaires : (L1 : < 3 méta ; L2 : multiples, toutes $<2 \mathrm{~cm}$; L3 : multiples, une ou plus $>2 \mathrm{~cm}$ ) - hépatiques, cérébrales, osseuses

Rapport présenté au $8^{\text {tme }}$ congrès de la SALF, Sousse Sept. 1990 b) Cette classification repose sur un bilan d'extension

- clinique

- marqueurs : béta HCG, AFP, LDH

La beta HCG et l'AFP sont des marqueurs de grande valeur pour le diagnotic positif devant un gros testicule. Ils sont élevés dans $80 \%$ des cas. La beta HCG peut être augmentée modérément dans les séminomes, mais toute élévation de l'AFP dénote la présence de TNS.

- imagerie : scanner abdomino-pelvien; lymphographie (facultative); scanner thoracique.

\section{II -Stade clinique I}

A/ Attitude classique : chirurgie première

I - Castration avec curage ganglionnaire

Le curage montre une atteinte clinique dans 20 à $30 \%$ des cas. $12 \%$ des $\mathrm{N}$ - et $50 \%$ des $\mathrm{N}+$ vont rechuter, mais seront guéris dans près de $100 \%$ par une chimiothérapie de rattrapage (3).

2 - Surveillance après castration sans curage Stratégie inaugurée à la fin des années 1970 (5). Un bilan peut maintenant en être établi.

- une totalisation de 7 séries (340 patients) en 1988 montre $27 \%$ de rechutes, $90 \%$ survenant dans les 12-18 premiers mois, $4 \%$ par an ensuite (2-4 ans), détectées dans $75 \%$ par les marqueurs; une guérison par chimiothérapie dans $96 \%$ de ces rechutes (qui sont détectées à un stade de maladie minime).

- une totalisation plus récente en 1990 (3) portant sur 680 patients, retrouve, avec un recul médian de 4 ans, $26 \%$ de rechutes (16-33\%) et une guérison par chimiothérapie dans $98 \%$ des cas.

3-Evaluation de ces 2 modalités

a) Le curage

- Permet une classification plus fiable, mais présente des risques de troubles de l'éjaculation et de stérilité. Des progrès techniques permettent toutefois de les réduire beaucoup (2). Il est inutile dans 70 à $80 \%$ des cas et est assorti d'un risque de $20 \%$ de rechutes.

b) La surveillance

- Evite le curage et ses risques, avec un taux équivalent de rechutes. Elle nécessite une surveillance stricte, clinique, biologique et d'imagerie (mensuelle la première année, bimensuelle la seconde année) et nécessite une très bonne adhérence des patients à cette stratégie.

c) Les résultats à long terme sont équivalents puisque, grâce à la chimiothérapie, la guérison est obtenue dans près de $100 \%$ des cas.

B/ Chimiothérapie adjuvante pour les patients avec facteurs de risque (invasion vasculaire ; tumeur indifférenciée ; stades localement avancés: T2 (au delà de l'albuginée, ou intra épididymaire); T3 (invasion du cordon spermatique ; T4 (envahissement du scrotum).

Il s'agit d'une stratégie récente pour laquelle nous ne disposons pas encore de résultats évaluables (3). Un essai est en cours au Medical Research Council, pour ces patients à risque, entre chimiothérapie adjuvante et surveillance (6).

\section{III - Stades IIA et IIB : 3 options.}

$1 /$ Chirurgie première avec curage

$50 \%$ des patients vont rechuter ensuite et seront rattrapés par une chimiothérapie seconde (3). Toutefois le taux de rechute est nettement plus faible pour les stades $A$.

$2 /$ Chimiothérapie adjuvante après chirurgie Cette stratégie a été testée par le Testicular Cancer Intergroup Study (9). 195 patients réséqués ont été randomisés entre pas de traitement et chimiothérapie par PVB. La survie est identique (98$99 \%$ ) dans les 2 groupes ( $50 \%$ des patients traités par chirurgie seule ont rechuté).

\section{3/ Chimiothérapie sans curage}

Deux séries de 50 patients montrent des taux de survie sans rechute très élevés (5).

\section{IV - Les stades avancés (IIC, III, IV)}

Leur traitement est dominé par la chimiothérapie. Dans ce domaine d'énormes progrès ont été enregistrés. Avant 1970, on ne disposait que de monochimiothérapie donnant des taux de rémission complète (RC) faibles de l'ordre de 10-20\%. Aux débuts des années 1970, la combinaison synergique Velbé-Bléomycine permet d'enregistrer des taux de $50 \%$ de RC. Mais le grand toumant se situe en 1974 avec la découverte de l'activité remarquable du platinum sur le cancer du testicule et encore accrue par son association au Velbé et à la Bléomycine. Les protocoles de référence sont alors le PVB (instauré par l'Indiana University Hospital) et le VAB (II à VI) (du Memorial Sloan Kettering Cancer Center de New York) et permettent d'obtenir 75 à $80 \%$ de RC. Le PVB (Platinum, Velbé, Bléomycine) est distribué par cycles de 5 jours pour 3-4 cycles espacés de 21 jours en traitement d'attaque. Le VAB6 (Cyclo- 
phosphamide, Velbé, Dactinomycine, Bléomycine, Platinum) est donné sur 4 jours pour 3-4 cycles espacés de 28 jours en traitement d'attaque. Un traitement d'entretien (essentiellement par Velbé) était donné initialement sur deux ans. Plus récemment le VP16 a remplacé dans de nombreux groupes le Velbé (protocole PEB) avec une même efficacité et une moindre toxicité comme l'ont montré des essais randomisés et le PEB est devenu le protocole de référence.

a) Résultats globaux obtenus par la chimiothérapie - La série du Royal Marsden Hospital (5) porte sur 320 patients traités entre 1976 et 1985 et enregistre à 5 ans $83 \%$ de patients en survie sans rechute (SSR) et constate que $90 \%$ des rechutes surviennent dans la première année.

- La série de l'Indiana University Hospital (7) porte sur 229 patients avec une médiane de follow up de 8,5 ans. La probabilité de survie à 12 ans est de $65 \%$; la probalité de SSR pour les RC est de $83,5 \%$ (les risques de rechute après $\mathrm{RC}$ sont donc faibles).

b) La chirurgie après chimiothérapie d'induction La chirurgie a une place dans le traitement de ces cancers avancés comme chirurgie de second look. Des masses résiduelles détectées par l'imagerie abdominale et/ou thoracique sont constatées dans $25 \%$ des cas et doivent faire l'objet d'une vérification chirurgicale qui constate un tératome mature dans $45 \%$ des cas, de la nécrose ou de la fibrose dans $40 \%$ des cas, et du cancer persistant dans $15 \%$ des cas nécessitant 2 cycles supplémentaires de chimiothérapie (5).

Au total, le pourcentage de guérison de ces cancers avancés le plus souvent métastasés est de l'ordre de $70-80 \%$, ce qui en fait un modèle de cancer curable.

\section{c) Résultats en fonction des facteurs de risque}

Toutes les grandes séries ont identifié le fait que le pronostic était dépendant du volume tumoral.

\section{Staging des groupes de risque}

a) Indiana University (4)

3 groupes (Groupe 1 : extension minimale ; Groupe 2 : extension modérée ; Groupe 3 : extension avancée) en fonction de l'importance de la masse abdominale et de l'importance de l'extension viscérale. Les taux de réponse sont respectivement de $99 \%, 91 \%, 53 \%$.

\section{b) Medical Research Council}

3 groupes de risques en fonction du volume tumoral et du taux des marqueurs. Les pourcentages de survie à 3 ans sont respectivement de $91 \%, 92 \%, 47 \%$.

\section{c) Autres systèmes}

Citons celui de Droz (Villejuif), modèle mathématique fondé sur le taux des marqueurs.

\section{Groupe de mauvais pronostic}

- Groupe minoritaire. Avec les protocoles conventionnels, les taux de contrôle de la maladie ne dépasse pas $50 \%$. Les résultats des tentatives d'amélioration des résultats par modification de protocoles de chimiothérapie sont plutôt décevants. La place de l'intensification par chimiothérapie à haute dose sous couvert d'autogreffe ne peut encore être évaluée.

\section{Groupe de bon pronostic}

Les pourcentages de guérison, on l'a vu, sont près de $100 \%$. Une désescalade thérapeutique est posssible. C'est ainsi que des essais randomisés ont montré qu'on pouvait réduire le nombre des médicaments (PE : PEB ou VAB6) et la durée du traitement (3 PEB : 4 PEB) (4).

$\mathrm{Au}$ total, des progrès fantastiques ont été obtenus par la chimiothérapie dans les formes avancées de cancer du testicule avec une guérison sans séquelies importantes et cela pour une grande majorité de patients. Toutefois il reste une ombre sur le pronostic des cancers du testicule : $20-30 \%$ des patients avancés ne sont pas mis en RC et $10 \%$ rechutent après RC.

\section{Les traitements de sauvetage}

Les drogues de sauvetage (Etoposide et Ifosfamide en particulier) permettent d'obtenir des taux de RC autour de 50\% mais la moitié seulement sont durables. La chimiothérapie à haute doses + autogreffe n'a une certaine efficacité que dans les cas où persiste encore une chimio-sensibilité.

\section{LES SEMINOMES}

- Se présentent presque toujours à un stade précoce (5)

Stade I : $77 \%$; stade II : $21 \%$; stade III-IV : $3 \%$

- Et ont une très grande sensibilité à la radiothérapie

\section{I - Stade I (5)}

Le traitement radiothérapique (prophylactique sur les aires ganglionnaires abdominales à la dose de 35-40 grays) permet d'obtenir la guérison avec un taux très faible de rechute $(2 \%)$.

\section{II - Stade II}

1/ IIA - IIB : Le traitement de référence reste la radiothérapie (40 grays, ganglionnaire abdominale) mais les taux de rechute sont plus grands (IIA : $10 \%$;IIB : 18\%). Il n'y a pas d'intérêt à une radiothérapie prophylactique sus diaphragmatique. 2/ IIC : Le taux de rechutes après radiothérapie trop élevé (38\%) fait préférer la chimiothérapie.

\section{III - Stades avancés}

La notion récente est que les séminomes sont aussi sensibles à la chimiothérapie que les TNS. Deux séries récentes montrent des taux de survie sans rechute de $80-85 \%$ (5).

La toxicité au long cours $(1,7)$

1/ Rénale (diminution de la fonction rénale) liée au platinum est minime, surtout à la phase d'induction, stable ensuite.

2/ Neurologique (neuropathie périphérique, atteinte ORL) liée au Velbé et au platinum s'est trouvée beaucoup réduite depuis l'abandon des traitements d'entretien.

3/ Il en est de même de l'atteinte vasculaire (syndrome de Raynaud surtout) et de l'atteinte pulmonaire (diminution de la capacité de diffusion du $\mathrm{Co}$ ) liée à la Bléomycine.

4/ Les leucémies secondaires induites par la chimiothérapie sont exceptionnelles.

5/ La spermatogénèse et la fertilité (3)

- Avant traitement, on note une réduction dans 50 à $70 \%$ des cas, et une impossibilité de stockage du sperme dans les $2 / 3$ des cas.

- Après traitement : on constate une azoospermie immédiate dans $100 \%$ des cas ; une récupération à 3-5 ans dans 60-80\% des cas.

- La possibilité de paternité avec des enfants bien portants et sans malformation congénitale.

6/ Impact psychologique

La qualité de la vie sexuelle n'est diminuée que dans $40 \%$ des cas et $90 \%$ des patients retrouvent un emploi à temps complet.

\section{REFERENCES}

1. Boyer M., Raghavan D., Harris PJ, Lietch J., Bleasel A., Walsh JC, Anderszon S., Tsang Chi S. Lack of late toxicity in patients treated with Cisplatin cotaining combination chemotherapy for metastatic testicular cancer. J. Clin. Oncol., $1990,81: 21-26$

2. Donohue JP. Preservation of ejaculation following nerve sparing retroperitoneal lymphadenectomy. J. Urol., 1988, 139 : $206 \mathrm{a}$

3. Gressler VH, Levine LA, Vogelzang NJ. A third option in the management of patients with clinical stage I Non Seminomatous Germ Cell Tumor. J. Clin. Oncol., 1990, $8: 4-8$ 4. Loehrer PJ, Williams SD, Einhorn LH. Testicular Cancer : the quest continues. J, Nat]. Cancer Inst., $1988 ; 80: 1373-1382$ 5. Peckham M. Testicular Cancer. Reviews in oncology 1 (1988) $\mathrm{N}^{\circ} 1$ (in Acta Oncologica, Vol 27, Fasc.4)

6. Pont J, Hołt N, Kosal D, Machacek E., Kienzer H., Sulcker H., Honetz N. Risk adapted choice in stage I non seminomatous lesticular germ cell cancer by regarding vascular invasion in the primary tumor. J. Clin. Oncol, 1990;16-20

7. Roth BJ, Greisl A, Kubilis PS., Williams SD., Einvoorn LH. Cisplatin based combination chemiotherapy for disseminated germ cell tumors : long term follow up. J. Clin. Oncol., 1988; 6: $1239-1247$

8. Stibley L, Brown M, Schuttinga A., Rothenberg M., Whalem J. Cis platin based combination chemotherapy in the treatment of advanced - stage testicular cancer : cost benefit analysis. J. Natl. Cancer Inst., $1990 ; 82: 186-192$

9. Williams SD, Stablein DM, Einhorn LH et al. Immediate adjuvant chemotherapy versus observation with treatment at relapse in pathological stage II testicular cancer. N. Eng. J. Med., 1987; 317 : 1433-1438

RESUME : Des progrès considérables ont été accomplis dans le traitement des cancers du testicule depuis une quinzaine d'années. Pour les tumeurs non séminomateuses de stade précoce (I et II) les taux de guérison avoisinent les $100 \%$ du fait de l'efficacité de la chimiothérapie de rattrapage des rechutes. Pour les stades cliniques $I$, la tendance est à la surveillance après castration sans curage. Pour les stades avancés métastasés, l'apparition du platinum a révolutionné les performances de la chimiothérapie qui permet de guérir 75 à $80 \%$ de ces patients. Il reste à améliorer les résultats d'un groupe de mauvais pronostic à forte masse tumorale. Les séminomes à un stade localisé (cas le plus fréquent) sont guéris par la radiothérapie; à un stade avancé, ils se montrent aussi sensibles à la chimiothérapie que les tumeurs non séminomateuses. Mots clés : séminome, tumeur non séminomateuse, chimiothérapie, radiothérapie, chirurgie, prognostic, stade. Andrologie, 1991, 1: 32-33 